\title{
A RARE CASE OF PRIMARY MALIGNANT MELANOMA OF THE VAGINA IN A YOUNG LADY: A REPORT
}

Sangeeta Pankaj ${ }^{1}$, Vijayanand Choudhary ${ }^{2}$, Rajesh Kumar Singh ${ }^{3}$

\section{HOW TO CITE THIS ARTICLE:}

Sangeeta Pankaj, Vijayanand Choudhary, Rajesh Kumar Singh. "A rare case of primary malignant melanoma of the vagina in a young lady: a report". Journal of Evolution of Medical and Dental Sciences 2013; Vol2, Issue 42, October 21; Page: 8146-8149.

\begin{abstract}
Malignant melanoma of vagina is a rare tumor with incidence rate of only 0.46 cases per one million women per year and less than 250 cases have been reported in medical literature till date. It has a notoriously aggressive behavior and is associated with a high risk of local recurrence and distant metastasis. At present, there are various treatment options for this disease but no standard guideline. It accounts for less than $1 \%$ of all malignant melanoma and less than $3 \%$ of all primary malignant tumors of the vagina. Conventional imaging techniques i.e. computed tomography, may be sub-optimal in evaluating a small volume of tumor, which may lead to inaccurate staging of a loco-regional tumor. The optimal treatment for vaginal melanoma is a subject of debate. Malignant melanoma of the vagina is a disease of postmenopausal women, with $75 \%$ of women being more than 50 years of age. Here, we present a case of primary vaginal malignant melanoma in a 35 year woman located in lower $1 / 3^{\text {rd }}$ of the vagina.
\end{abstract}

KEY WORDS: Malignant Melanoma, Vagina, Melanocytes.

INTRODUCTION: Malignant melanoma is an aggressive disease which affects both skin and mucosal surfaces. Melanoma of the vagina and urethra is an extraordinarily rare tumor accounting for less than $10 \%$ of all female genital tract melanomas, and $2.6 \%$ of all vaginal cancers [1]. Malignant melanoma of the vagina is a disease of postmenopausal women with $75 \%$ of women being over 50 years of age [2]. It may arise anywhere in the vagina with a predilection for the lower third [1]. Due to the scarcity of cases of malignant melanoma of the vagina, there is little evidence-based literature for guiding clinicians to understand the importance of disease staging via noninvasive imaging strategy. This is a contentious issue which affects the way this tumour is managed appropriately, whereby tumour size, regional infiltration and groin lymphatic involvement are the cornerstone for disease prognostications $[3,4]$.

CASE REPORT: A 35 year old woman, Para 3, post-surgical menopause by 3 years, had undergone hysterectomy for Dysfunctional Uterine Bleeding. She presented in gynecological oncology department with complaints of white discharge and recurrent bleeding per vaginum for last 14 months. Per speculum examination revealed, two blue to brown coloured lesion measuring 2 $\mathrm{cm}^{2}$ each, in the lower $1 / 3$ rd of the vagina placed $3 \mathrm{cms}$ apart, on the postero-lateral wall of vagina. Another $1 \mathrm{~cm}^{2}$ lesion was found below the urethra. Around two third of vaginal wall was having a bluish flush. On per vaginal examination uterus was not palpable and both the adnexa was also not palpable. Rectum was normal and there was no inguinal lymphadenopathy. Wide excision biopsy (with free margin of $1 \mathrm{~cm}$ ) was done from all the three lesions and sample sent for histopathological examination. 
MRI pelvis revealed a $1.8 \times 1.6 \mathrm{~cm}$ ill defined heterogenous enhancing lesion in the lower end of vagina which was also involving the external urethral meatus. Rest of the vagina, urethra, bladder and rectum showed normal morphology and no lymphadenopathy. On cystoscopy, external meatus was found to be indurated but bladder and urethra was normal. Sigmoidoscopy was normal.

Histopatological report diagnosed the tumor as malignant melanoma involving the vaginal mucosa. The tumour was composed of epithelioid cells arranged in irregular sheets invading the submucosal stroma diffusely. These cells had large pleomorphic nucleus and prominent nucleoli. The cytoplasm was scanty to moderate in amounts. The cytoplasm contained abundant golden brown granular melanin pigment. Melanin pigment was also present extra-cellularly.

The patient was treated with external beam radiation (40 Gy in 20 fractions; 5 fractions per week) with palliative intent, to the tumor bed, followed by chemotherapy with six cycles of Temozolomide, $250 \mathrm{mg}$ orally from D1 to D5 three weekly for 6 cycles. The patient is alive after 1 year and is under follow up.

DISCUSSION: Malignant melanoma of the vagina is a very rare tumor, accounting for $0.3-0.8 \%$ of all melanomas in women and 2 - 5\% of female genital tract melanomas, and less than $3 \%$ of vaginal tumors. [5] The tumor typically presents in the sixth to seventh decade of life and occurs more commonly in the lower third of the vagina and involves the anterior wall of vagina.[5,6] The appearance of tumors is almost always pigmented with only $10-20 \%$ being amelanotic. [7] The most common symptom is abnormal vaginal bleeding. [6].

Grossly, the tumor appears as black or grayish black nodular, polypoid or fungating mass. Frequently, the overlying epithelium is eroded or ulcerated which explains their frequent confusion with squamous cell carcinoma. The differential diagnosis includes metastasis from other sites, poorly differentiated squamous cell carcinoma, sarcoma, lymphoma and blue nevus.

Several treatment options are administered but none of them are considered to be a standard approach. Surgical resection is considered the treatment of choice with survival benefits. $[6,7]$ Different surgical methods such as wide local excision, radical surgery (total vaginectomy with or without vulvectomy), and pelvic exenteration have been described. However, research has continued to demonstrate that there was no difference in survival between patients who have radical surgical procedures and those who have more conservative surgery.[8,7,9] Some authors advise for radical surgery due to the aggressive behavior of vaginal melanoma.[8,9] After surgery, patients with a high risk of recurrence (including regional lymph node involvement or thicker primary tumors, i.e. American Joint Committee on Cancer Stage IIb /IIc / III) should be considered for adjuvant therapy.

The role of cytotoxic chemotherapy in vaginal melanoma has not been completely defined because of the small number of cases. Dacarbazine has been considered the standard of treatment for metastatic or recurrent melanoma since 1972. Other useful antineoplastic agents include temozolomide, platinum analogs, nitrosoureas, and taxanes. The response rate of these single agents is $11-22 \%$, with median overall survival of 5.6-11 months. Combination chemotherapy that is most commonly used for melanoma includes Dartmouth regimen (Dacarbazine/ Carmustine/Cisplatin/Tamoxifen) and CVD regimen (Cisplatin/Vinblastine/Dacarbazine), which have proved to increase objective response rate but have no overall survival benefit.[10] Based upon 
the above observations, it may be possible to develop combination chemotherapy regimens that improve overall survival in near future.

Radiotherapy for local control has been mostly offered in the following two conditions, surgically non-resectable disease or as an adjuvant therapy in case of pathologically positive margins or positive lymphadenopathy. [6, 7] Prognosis is poor with a 5-year survival rate ranging from $10-20 \%$. It appears to be influenced by tumor size with lesions greater than $3 \mathrm{~cm}$ having a bad prognosis. Age, mitotic count, stage and location of the lesion do not influence survival.

To conclude, early diagnosis of a loco-regional malignant vaginal melanoma may give patients the best chance for prolonged survival. The most accepted line of treatment of malignant melanoma of the vagina is surgery followed by post-operative radiotherapy. Primary malignant melanoma of the vagina has a poor prognosis with high risk of local recurrence and distant metastasis. In our case, local excision followed successively by radiotherapy and chemotherapy proved to be a tolerable modality.

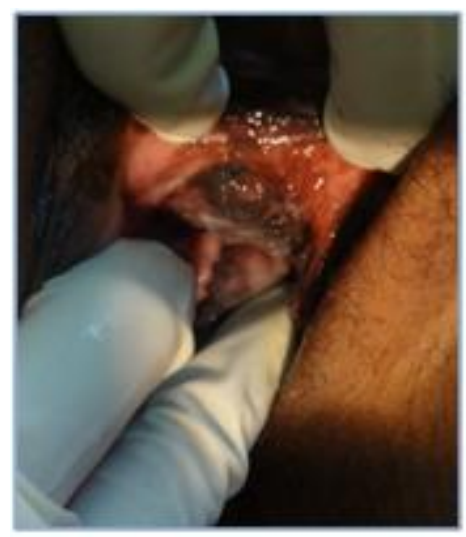

Fig.1: Vagina showing blue to brown discoloration of the vagina and the lesion below urethra.

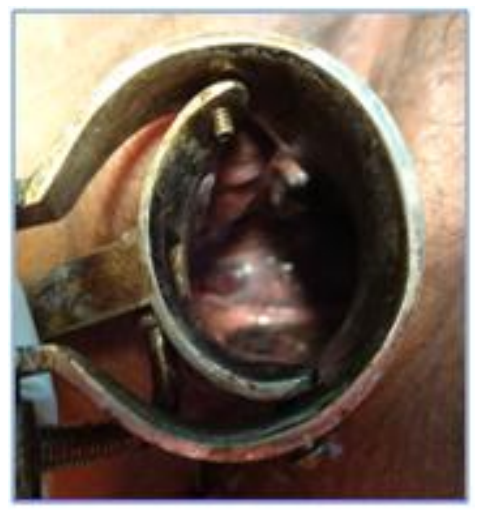

Fig.2: Vagina showing brown discoloration of vagina and lesion on lower third of vagina.

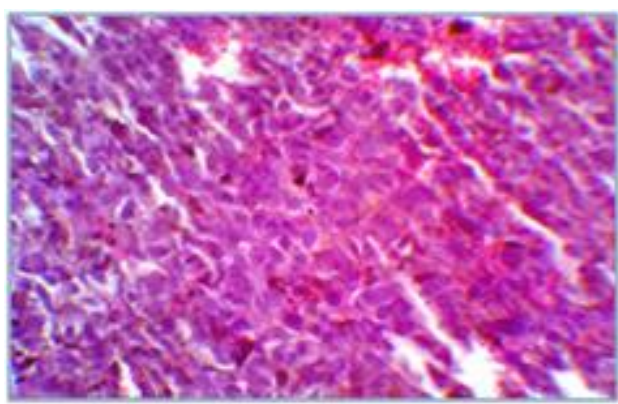

Fig. 3: Numerous atypical melanocytes are seen arranged in irregular sheets. Golden brown granular melanin pigment is present both intracellularly and extracellularly. 


\section{REFERENCE:}

1. Moros M L, Ferrer F P, Mitchell M J, Romeo J A and Lacruz R L. Primary malignant melanoma of the vagina: poor response to radical surgery and adjuvant therapy. Eur J Obstet Gynecol Reprod Biol 2004; 113(2):248-250.

2. Parikh JH, Barton DP, Ind TE and Sohaib SA. MR imaging features of vaginal malignancies. Radiographics 2008; 28(1):49-63.

3. Tjalma WA, Monaghan JM, de Barros Lopes A, Naik R and Nordin A. Primary vaginal melanoma and long-term survivors. Eur J Gynaecol Oncol 2001; 22(1):20-22.

4. Stehman FB and Look KY. Carcinoma of the vulva. Obstet Gynecol. 2006; 107(3):719-733.

5. Samolis S, Panagopoulos P, Kanellopoulos N, Papastefanou I, Karadaglis S, Katsoulis M. Primary malignant melanoma of the vagina: case report. Eur J Gynaecol Oncol 2010; 31:2334.

6. Michael F, Mariano E, Charlotte CS, Pamela TS, Patricia JE, Charles FL, et al. Primary malignant melanoma of the vagina. Obstet Gynecol 2010; 116:1358-65.

7. Miner TJ, Delgado R, Zeisler J, Busam K, Alektiar K, Barakat R, et al.

8. Tjalma WA, Monagham JM, de Barros Lopes A, Naik R, Nordin A. Primary vaginal melanoma and long-term survivors. Eur J Gynaecol Oncol 2001; 22:20-2.

9. Buchanan DJ, Schlaerth J, Kurosaki T. Primary vaginal melanoma: thirteen-year disease-free survival after wide local excision and review of the literature. Am J Obstet Gynecol 1998; 178:1177-84.

10. Lens MB, Eisen TG. Systemic chemotherapy in the treatment of malignant melanoma. Expert Opin Pharmacother 2003; 4:2205-11.

\section{AUTHORS:}

1. Sangeeta Pankaj

2. Vijayanand Choudhary

3. Rajesh Kumar Singh

\section{PARTICULARS OF CONTRIBUTORS:}

1. Assistant Professor, Department of Gynaecological Oncology, RCC, Indira Gandhi Institute of Medical Sciences, Patna.

2. Assistant Professor, Department of Pathology, Indira Gandhi Institute of Medical Sciences.

3. Associate Professor, Department of Gynaecology, RCC, Indira Gandhi Institute of Medical Sciences, Patna.

NAME ADDRESS EMAIL ID OF THE CORRESPONDING AUTHOR:

Dr. Rajesh Kumar Singh, Associate Professor, RCC, Indira Gandhi Institute of Medical Sciences, Patna.

Email-drrajeshsingh@yahoo.com

Date of Submission: 04/10/2013.

Date of Peer Review: 05/10/2013.

Date of Acceptance: 17/10/2013.

Date of Publishing: 19/10/2013 